\title{
Innovation Design Research on the Apparel Tag from the Perspective of Advanced Design Technology
}

\author{
WU HUANG ${ }^{1, \mathrm{a}}$, LU JINSHENG $^{* 1, \mathrm{~b}}, \mathrm{YU}_{\mathrm{CHENGCHENG}}{ }^{1, \mathrm{c}}$ \\ ${ }^{1}$ College of Mechanical Engineering Donghua University Shanghai, China
}

\begin{abstract}
The visual communication design of the apparel tag is essential to ensure a good interaction mechanism between the consumer, the clothing and the enterprise. However, there is a serious phenomenon of homogenization, which shows that the apparel tag lack of innovation design. By analyzing the information displayed, the apparel tag is not only the carrier of information transmission but also the presentation of brand culture. With the development of advanced design technology, it is proposed that the apparel tag should be designed from the three orientations of personalized design, intelligent design and green design, which can completely realize the function of the apparel tag.
\end{abstract}

\section{Introduction}

With the improvement of living standards, people pay more attention to the appearance, so that the clothing industry has been rapid development. However, the apparel tag has not developed accordingly. At present, some clothing enterprises focus on the design and production of clothing, but they ignore the innovation design of the clothing tag. A relevant study found that apparel tags could influence consumers' purchase intentions and also enhanced their positive attitudes toward apparel brands 1 . There is a serious phenomenon of homogenization, which hinders the development of clothing tag to a certain extent 2 . As an indispensable part of clothing, the apparel tag can accurately express relevant information of clothing in a limited space. Regardless of any brands, the apparel tag has become a necessary accessory for every piece of clothing, so the innovation design of the apparel tag has a great potential market. Therefore, how to improve the innovation design ability of the apparel tag is an important subject at present.

\section{Functions of the Apparel Tag}

The apparel tag is the instruction of clothing products in the form of hanging, which contains clothing type, manufacturer information, composition and content, size, price, maintenance method, precaution, etc. Usually, the apparel tag is composed of the nameplate, hanging grain and hanging wire. Some are attached with material blank or accessories like buttons. Among them, the common materials of nameplates are paper, metal, plastic and natural materials. As the identity of clothing, the apparel tag has become an indispensable part of the composition of the whole clothing.

\footnotetext{
awoohuang@foxmail.com

b*lujinsheng888@126.com

c1720842436@qq.com
}

\subsection{Carrier of Information Transmission}

As a carrier of information transmission, the apparel tag has the basic function of conveying clothing information. Tab. 1 shows the common contents and functions on the apparel tag.

Table1. Content and function of apparel tags

\begin{tabular}{|c|l|}
\hline Content & \multicolumn{1}{|c|}{ Function } \\
\hline Clothing type & It indicates the type of clothing. \\
\hline $\begin{array}{c}\text { Manufacturer } \\
\text { information }\end{array}$ & $\begin{array}{l}\text { It mainly includes the manufacturer's } \\
\text { name and address to identify the } \\
\text { subject of legal liability. }\end{array}$ \\
\hline Specification & $\begin{array}{l}\text { It indicates the applicable scope of } \\
\text { length and size. }\end{array}$ \\
\hline Price & It indicates the value of clothing. \\
\hline $\begin{array}{c}\text { Fiber composition } \\
\text { and content }\end{array}$ & $\begin{array}{l}\text { It indicates raw material and its } \\
\text { composition. }\end{array}$ \\
\hline
\end{tabular}

The apparel tag accompanies the whole life cycle of clothing and plays a corresponding role in every sales link of clothing including before, during and after sales. For merchants, they can check models, manage inventory and track sales status according to the apparel tag. It is convenient to stock and sell clothes to realize information management. For consumers, they have a practical orientation when they use apparel tags 3. Identifying clothing information through the apparel tag is the most direct and quickest way according to their needs to buy clothes. In addition, the apparel tag is the voucher for return and replacement to provide support for the maintenance of clothing, which is the embodiment of the rights of consumers. 


\subsection{Presentation of Brand Culture}

The apparel tag that conveys commodity information is related to the shaping of brand culture closely. With the increasing prosperity of the clothing market, the apparel tag that can directly communicate with consumers has become a way to shape the brand terminal image of the enterprise. The apparel tag is not only the retail material of clothing but also the symbol of clothing brand culture. As an indispensable part of the Visual Identity of enterprises, the apparel tag is an effective carrier of brand identity. It can directly affect consumers' cognition, so it is of great significance for enterprises to brand promotion and cultural publicity 4 .

In order to promote the brand culture, enterprises constantly emphasize the brand image through the apparel tag. The patterns, symbols and colors on the apparel tag correspond with the brand culture, giving consumers visual stimulation for leaving the brand impression and increasing the brand recognition.

\section{Design Elements of the Apparel Tag}

The text, the graphic and the color are important design elements on the apparel tag, which are the key to the effective communication of the apparel tag.

\subsection{Text Element}

The text is the main design element of the apparel tag and it is an important link to convey information. Due to the small size of the apparel tag, the presentation of text content should have a primary and secondary relationship. The text element can divide into numbers, languages (like Chinese and English), etc. In order to visualize, the text design whatever the form should consider font, font size, word spacing and line spacing. On the overall arrangement, taking into account the human visual system's habit of reading information, the layout should be arranged from top to bottom and from left to right.

\subsection{Graphic Element}

Compared with the text, the graphic has a variety of different forms of expression, so it has a strong antiinterference force and can convey more information. In the design of the apparel tag, graphic elements are primarily used to the manufacturer information and maintenance methods. As shown in Fig. 1, symbolic patterns are widely used, such as Playboy's rabbit and Boy London's eagle. Strong visual graphics can quickly seize the attention of consumers to guide consumers to purchase. Therefore, enterprises often place their logos in a conspicuous position on the apparel tag. Because standardized graphics are conducive to quick understanding for users, graphic symbols conforming to national standards such as washing standards should be used in the design of apparel tags. (a)

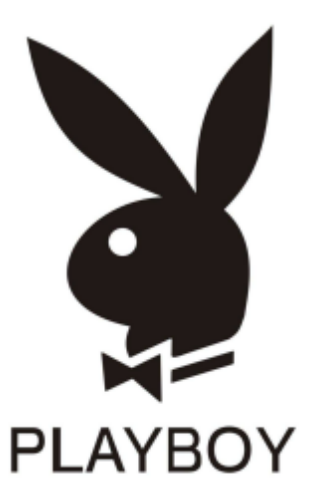

(b)

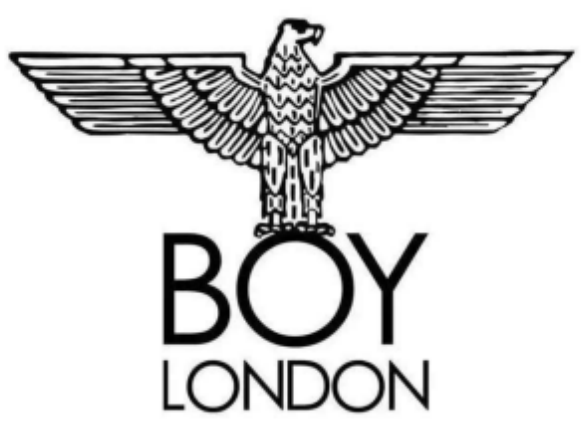

Fig1. Graphic elements.

\subsection{Color Element}

Color is also an important design element of the apparel tag. In the use of color, it is necessary to comprehensively consider the apparel tag, the clothing itself and the use environment, which complement and restrict each other. The color representing the brand image is applied to each clothing tag. Consumers can judge and identify the clothing brand through color, which can increase brand loyalty.

As shown in Fig. 2, taking Li-Ning, a Chinese famous brand, for example, the main colors on the apparel tag of different series clothing all adopt the brand colors-LiNing red and Li-Ning white. Fig. 2(a) shows a normal apparel tag, and Fig. 2(b) shows the apparel tag of the China Li-Ning series. All apparel tags of Li-Ning convey a modern and optimistic brand personality, giving consumers a strong sense of unity, so that greatly deepens the brand impression.
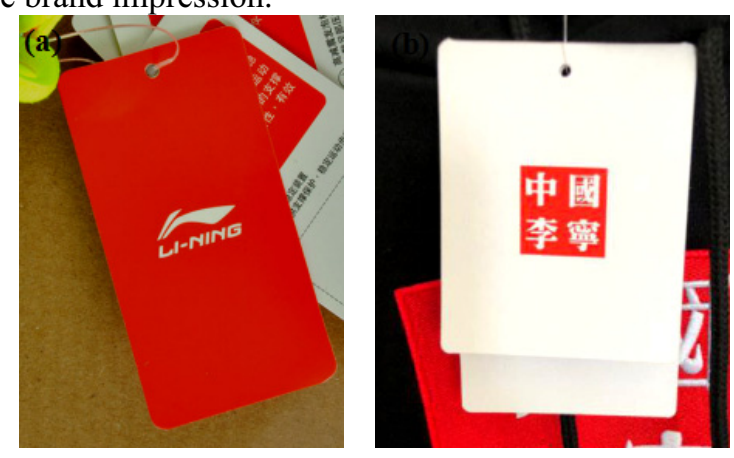

Fig2. Apparel tags of Li-Ning. 


\section{Innovation Design Strategy and Analysis of Apparel Tags}

\subsection{Personalized Design}

There is personalized in modern design gradually, and consumers constantly continue to favor the products that can express personal aesthetic and self-style. The design of the apparel tag can affect the sales of clothing to a certain extent. Enterprises could win the best position in the fiercely competitive market by the personalized design of the apparel tag because that can attract consumers. Therefore, more and more enterprises are aware of the function of the apparel tag that can promote brand culture and begin to pay attention to the continuous innovation of visual communication of the apparel tag. From simple to complex, the design presents diversification. The personalized design of the apparel tag requires complete functions not only to meet people's cognitive needs but also people's aesthetic needs. Therefore, personalized design tests the designer's thinking ability.

On the one hand is the personalized design on modeling. The conventional shape of the apparel tag is rectangular. With the maturity of die-cutting technology, the modeling presents a variety of forms, forming a new visual effect. As shown in Fig. 3(a), the birdcage shape of the apparel tag is novel and unique, which adds a sense of interest. In terms of modeling, the apparel tag could also design for consumers of different ages and genders with corresponding characteristics. As shown in Fig. 3(b), for children's clothing, cartoon images such as little monsters are more in line with the cognition of children. On the other hand is the personalized design on the material. Common apparel tags are made of a single material like paper, metal, plastic or natural material. While the personalized design on the material is no longer limited to the single material. The apparel tag in Fig. 4 is made of both kraft paper and non-woven fabric. Nowadays, the garment industry has gradually transformed from costdriven to innovation-driven. With the discovery of more and more new materials, unconventional materials such as fluorescence material and silica gel also been applied to the apparel tag.

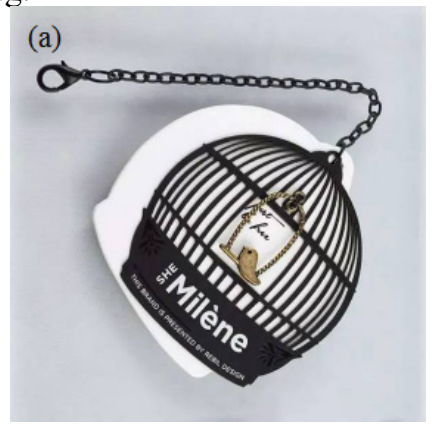

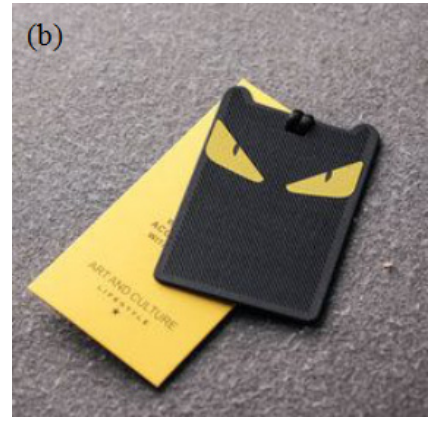

Fig3. Personalized design on modeling.

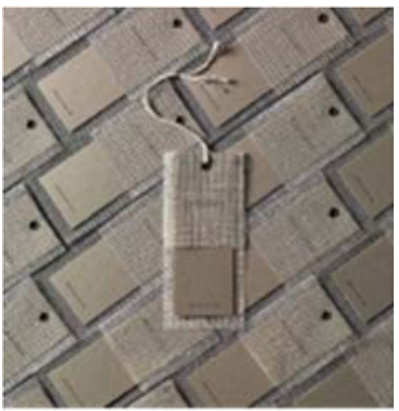

Fig4. Personalized design on the material.

\subsection{Intelligent Design}

The revolution of information technology has laid a foundation for intelligent products. The intelligent design integrates the high and new technologies like digital technology into one to solve problems that traditional technologies cannot solve. It could meet people's different needs and strictly control production processes. The combination of technology and the apparel tag plays an important role in improving the communication efficiency of the supply chain and the precise management of clothing. With the rapid development, increasing maturity and decreasing cost of technology, the intelligent design of the apparel tag has a broad space for development.

At present, the intelligent design of the apparel tag has made some progress and has been recognized by the industry. For example, Fig. 5 shows an apparel tag with Radio Frequency Identification (RFID) technology. RFID has the advantages of fast reading and writing speed, simple reading process and anti-collision. This is conducive to a more refined and automated management of clothing 5. Besides, the application of digital anticounterfeiting technology in the design of the apparel tag can prevent fake clothing to circulate in the market. Meanwhile, it can maintain the reputation and brand image of enterprises and protect the legitimate rights of consumers. 


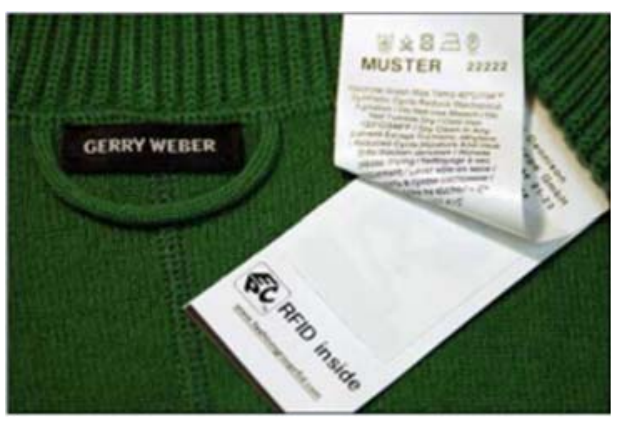

Fig5. An apparel tag with RFID.

\subsection{Green Design}

Green design is a kind of design concept that meets current needs without compromising the ability of future generations to meet their own needs. As a design concept of low carbon and environmental protection, green design idea applied into the design of modern products is the inevitable road of social development. The use time of the apparel tag that is the accessory of clothing is shorter compared with clothing. After buying clothing, consumers quickly discarded the apparel tag without reasonable utilization or recycling, which leads to the waste of resources and environmental pollution. With the development of society, all links of the garment industry are undergoing positive transformation, so the green design of the apparel tag is also an embodiment of meeting the requirements of the times. Moreover, with the increasing rationalization of the domestic consumption concept, consumers' cognitions and demands for environmental protection are becoming more and more specific 6 .

The design of the apparel tag should pay more attention to the design concept of green development, to realize the harmony and unity between products and the environment. Reducing production costs and the waste of natural resources, improving the utilization rate of resources, which are not only conducive to environmental protection but also to increase the added value of products. On the one hand, from the perspective of materials, using green materials such as degradable materials and rapidly recycled materials is the simplest and most direct green design for apparel tags. On the other hand, as shown in Fig. 6 , from the perspective of the product life cycle, apparel tags can be transformed into daily necessities, like coil winder, toy, calendar, bookmark and ruler, to extend the life cycle. It should promote secondary utilization and endue apparel tags with more functional value.

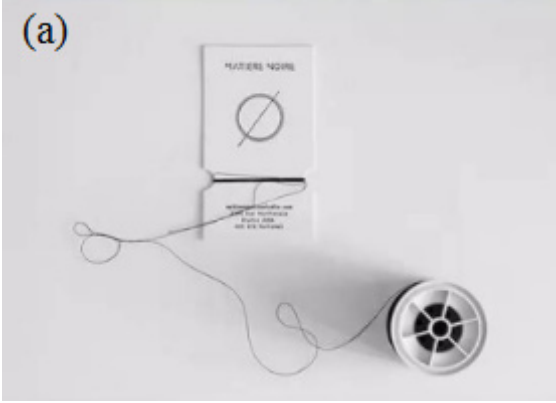

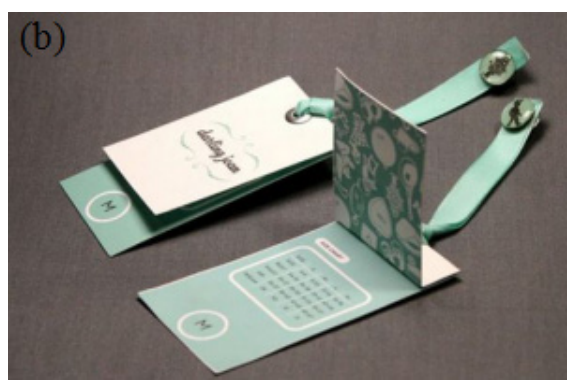

Fig6. Green design of the apparel tag. Figure 6(a) shows a coil winder. Figure 6(b) shows a calendar.

\section{Conclusion}

The development of the apparel tag depends on the development of the garment industry. The apparel tag is not a single individual but an important part of clothing. Designers should pay more and more attention to the innovation design of the apparel tag with the development of advanced design technology. Although the size of the apparel tag is small, it is the main way to convey information about clothing and promote brand culture as an advertising medium for enterprises. The design of the apparel tag covers many fields including visual communication, cognitive psychology and social economics. Innovating design of the apparel tag is conducive to form the core competitive advantage for enterprises and to have a positive effect on sales of clothing and brand culture communication.

\section{References}

1. Hyllegard, K. H., Yan, R. N., Ogle, J. P., Lee, K. H. (2012) Socially responsible labeling: the impact of hang tags on consumers' attitudes and patronage intentions toward an apparel brand. Cloth. Text. Res. J., 30: 51-66.

2. Perez, A., Lonsdale, M. (2018) Garment label design and companion information to communicate fashion sustainability issues to young consumers. J. Visual Lang. Comput., 52: 114-139.

3. Petrescu, D., Bran, F., Ioan, I., Rădulescu, C. V. (2016) Clothing label and ecological label: a missed opportunity or a powerful tool in the marketplace. J. Ind. Text., 67: 413-419.

4. Memon, N. (2018) Readymade garment industry: brand consciousness among people increasing every day. Pakistan Textile Journal, 68: 54-55.

5. Cilloni, G., Leporati, R., Rizzi, A., Romagnoli, G. (2019) State of the art of item-level RFID deployments in fashion and apparel retail. Int. J. RF Tech., 10: 65-88.

6. Ma, Q. F. (2019) Research on promoting sustainable development of textile and garment industry from the perspective of design. Int. J. Sci., 6: 64-69. 